\title{
3 Researchs buare \\ Two Cases of Anomalous Origin of Left Coronary Artery From The Pulmonary Artery
}

\section{Zhao Juan}

Taizhou People's Hospital, Taizhou

Zou Chunbo ( $\nabla$ shilz123@yeah.net )

Taizhou People's Hospital, Taizhou

\section{Case report}

Keywords: Cardiovascular malformations, coronary arteries, coronary CTA

Posted Date: October 27th, 2021

DOl: https://doi.org/10.21203/rs.3.rs-961625/v1

License: (c) (i) This work is licensed under a Creative Commons Attribution 4.0 International License. Read Full License 


\section{Abstract}

Coronary artery originating from pulmonary artery is a rare congenital vascular malformation, which generally presents corresponding clinical symptoms with the growth of patients' age. Coronary CTA and angiography are important methods for diagnosis of this disease, and provide evidence for treatment strategies of patients.

\section{Introduction}

The origin of the left coronary artery from the pulmonary artery is rare in clinical practice, and the treatment experience is relatively lacking. With the development of science and technology, medical imaging is timely and more advanced, which is beneficial for us to more accurately observe the malformed blood vessels and disease changes in patients. Here, we introduce two cases.

\section{Case Report}

Case 1: The patient, male, 53 years old,was admitted because of "chest tightness for 1 week",BNP120pg/ml. UCG showed abundant collateral blood flow in the ventricular septum(Fig.1A), and the left coronary artery was connected to the pulmonary artery. Blood flowed to the pulmonary artery in diastolic phase and pulmonary artery in systolic phase. (Fig.1B) (Fig.1C). Coronary angiography showed that the left coronary artery was absent and the right coronary artery was thick. (Fig.1D), (Fig.1E).

Coronary CTA showed that the left coronary artery was connected to the pulmonary artery (FIg. 1F). There were abundant collateral vessels in the interventricular septum (FIg. 1G, H, I). The posterior interventricular branch of the right crown was connected with the end of the anterior descending branch of the left crown (Flg. 1J). The right coronal conus branch and the anterior branch of the right ventricle are connected with the branches of the left anterior descending branch (Flg. 1K). The left coronary artery was diagnosed as originating from the pulmonary artery. The patient's symptoms spontaneously resolved after admission and surgical treatment was refused.

Case 2: A 46-year-old male patient was admitted to hospital due to " repeated chest tightness and asthma for 1 year and aggravation for 2 weeks after activities". ECG suggested atrial fibrillation, BNP8667pg/ml, UCG suggested abnormal coronary blood flow, left heart enlargement, left ventricular diastolic dysfunction (restrictive filling disorder), severe mitral regurgitation, moderate pulmonary hypertension, small amount of fluid in pericardium.

Coronary CTA indicated that the left coronary artery was connected with the pulmonary artery (Fig. 2A and $2 \mathrm{~B}$ ), and both the left and right coronary arteries were wide and malformed, with abundant collateral vessel formation between them. (Figure $2 \mathrm{C}, \mathrm{D}, \mathrm{E})$

Coronary angiography: left coronary artery was absent and right coronary artery were wide malformation. (Figure $2 F, G$ ), diagnosed as abnormal origin of coronary artery (left coronary artery originates from 
pulmonary artery), mitral valve replacement + left main trunk ligation + coronary artery bypass grafting were performed in our hospital.

\section{Discussion}

The left coronary artery originates from the pulmonary artery (ALCAPA) also known as bland-whitegarland syndrome is a rare congenital abnormality first reported by Brooks in 1886. [1] Approximately 1 in 300,000 live births. [2] The patient had no special discomfort in his childhood and young adulthood, but gradually presented with dyspnea, angina pectoris, syncope and other clinical symptoms as he grew older. Symptoms of ALCAPA are usually associated with myocardial ischemia. The lateral branch of the right coronary artery usually forms when pulmonary resistance drops and supplies blood to the left coronary artery to satisfy the oxygen consumption of the myocardium. If the pulmonary artery perfusion pressure is reduced, blood flow in the left coronary artery will enter the pulmonary artery which called " steal blood "phenomenon, leading to ischemia and hypoxia in left ventricular. Large shunts from left to right often result in chronic ischemia, even with adequate collateral circulation.

In many cases, the anterolateral papillary muscle may contract due to scarring and calcification from chronic ischemia, a process that may lead to mitral regurgitation. [3] Mitral valve replacement may be considered in patients with severe mitral valve prolapse. The second patient had mitral regurgitation, which was considered to be related to papillary muscle ischemia. After mitral valve replacement + left main stem ligation + coronary artery bypass graft, the patient's heart failure symptoms were significantly improved during a 3-year follow-up.

According to whether there is abundant collateral circulation between the left and right coronary arteries, the disease can be divided into infantile type and adult type. Because the pressure and oxygen saturation of the aorta and pulmonary artery in the fetus are equal, there are no obvious signs of disease during the fetus, and no collateral branches form during this time due to normal myocardial perfusion. However, after birth, pulmonary vascular resistance decreases and coronary artery theft occurs. The onset of symptoms depends on the number of collateral branches between the left and right coronary arteries and pulmonary artery pressure. [4] A review of ALCAPA patients showed that $66 \%$ of adult patients presented with angina, dyspnea, palpitations or fatigue. Ventricular arrhythmias, syncope, or sudden death occurred in $17 \%$ of patients, while $14 \%$ remained asymptomatic. [5]

Surgery and percutaneous closure are the main treatment methods for these patients. Surgical treatment includes:(1) ligation of the left main artery and reconstruction of the left coronary artery system with a vein or internal mammary artery graft (2) direct or indirect reimplantation of the left aorta to the aorta via a pulmonary artery or internal iliac artery graft. Ligation of the abnormal artery with coronary artery bypass grafting is the preferred method in adults, and reimplantation of the left main coronary artery into the aorta is the most common technique in the pediatric population. The main treatment for minimally invasive percutaneous treatment was percutaneous ALCAPA closure with amplazee vascular occluder. [6] 
Imaging examination is crucial for the differential diagnosis of ALCAPA patients. Early diagnosis combined with necessary surgical treatment can significantly improve the prognosis of these patients.

\section{Abbreviations}

CTA:CT angiography

UCG:Ultrasound cardiogram

ECG:Electrocardiogram

ALCAPA:Anomalous origin of the left coronary artery from the pulmomary artery

\section{Declarations}

Acknowledgements: None.

Authors' contributions $\rrbracket$

Z C B - acquisition of case and the preparation of the manuscript. Z J-planning and manuscript revision, interpretaion of results and making contributions to the conception of this study. All authors have read and approved this manuscript.

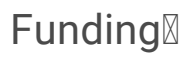

There was no funding for this study.

Availability of cases and materials $\Downarrow$

All information are available from the corresponding author on reasonable request.

Ethics approval and consent to participate $\mathbb{Z}$

This study has been granted an exemption from requiring ethics approval according to "The ethics committee of Taizhou People's Hospital".

Consent for publication $\rrbracket$

Obtained.

Competing interests $\rrbracket$

$\mathrm{Z} \mathrm{J}$ andZ C B declare that they have no competing interests.

\section{References}


1. BROOKS HS. Two Cases of an Abnormal Coronary Artery of the Heart Arising from the Pulmonary Artery: With some Remarks upon the Effect of this Anomaly in producing Cirsoid Dilatation of the Vessels [J]. J Anat Physiol, 1885, 20 (Pt 1):26-29.

2. Paone G, Silverman NA. Anomalous origin of the left coronary artery from the pulmonary artery [J]. The Journal of Thoracic Cardiovascular Surgery. 1994;108(6):1147.

3. LEE AC, YEGHIAZARIANS FOSTERE. Y. Anomalous origin of the left coronary artery from the pulmonary artery: a case series and brief review[J]. Congenit Heart Dis. 2006;1(3):111-5.

4. YAKUT K, TOKEL N K, OZKAN M, et al. Diagnosis and treatment of abnormal left coronary artery originating from the pulmonary artery: A single-center experience [J]. Anatol $\mathrm{J}$ Cardiol. 2019;22(6):325-31.

5. BUTT A, AMANULLAH MM, AMANULLAH MA, et al. Anomalous origin of the left coronary artery from the pulmonary artery: A surgical certainty [J]. Pak Med Assoc. 2020;70(3):561-4.

6. SUZUKI T, ITTLEMAN F P, GOGO P B, Jr. Atypical presentation of anomalous origin of the left main coronary artery from the pulmonary artery [J]. Ann Thorac Surg. 2009;88(4):1339-41.

\section{Figures}
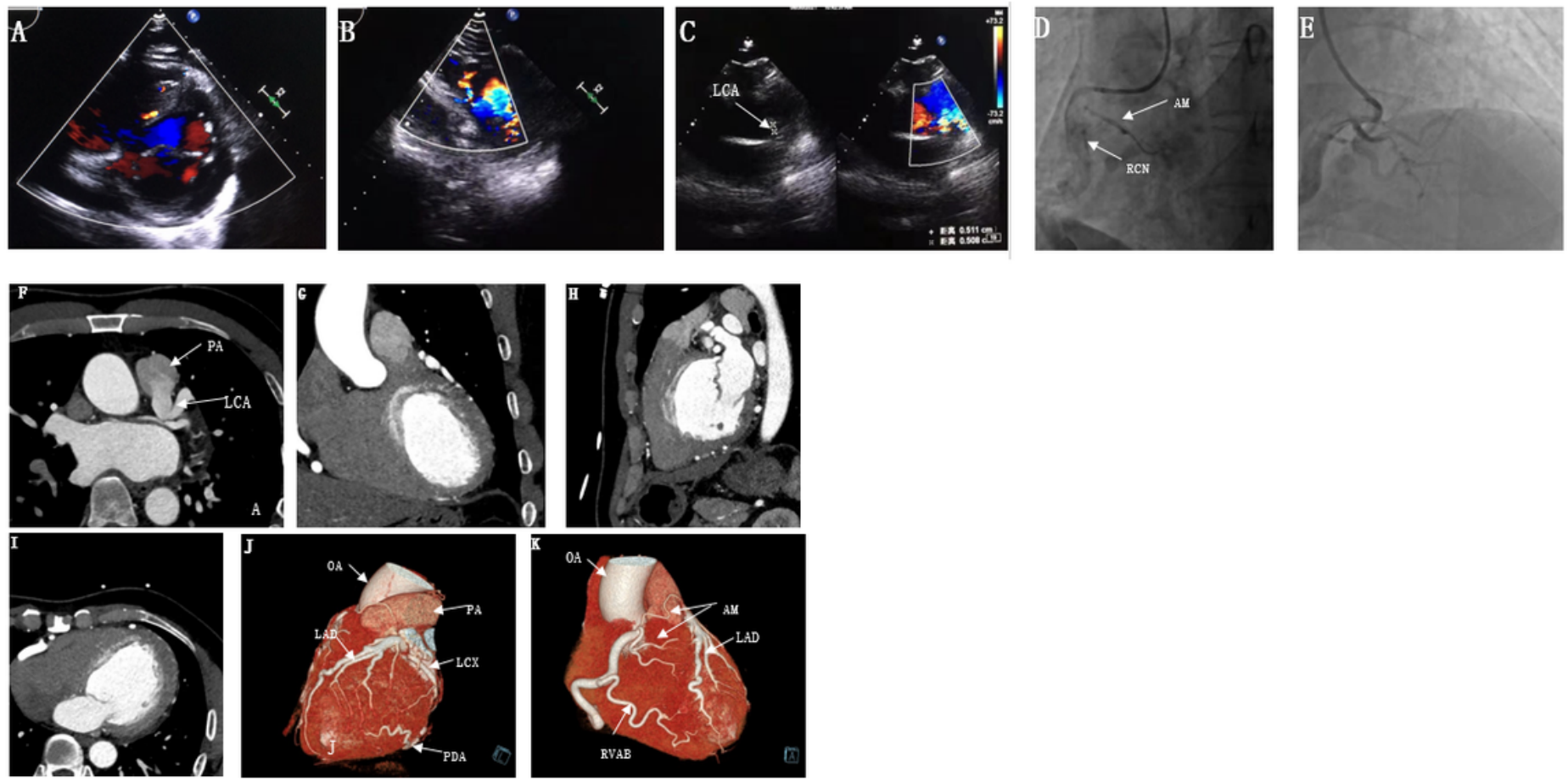

Figure 1

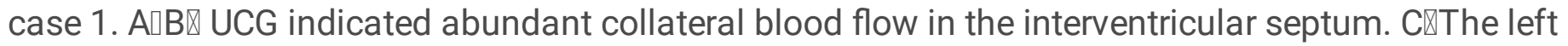
coronary artery is connected to the pulmonary artery. Diastolic blood flows to the pulmonary artery, and systolic pulmonary blood flows to the left coronary artery. case 1.D』EखCoronary angiography of the 
patient: the left coronary artery was absent and the right coronary artery was thick. case 1 . Coronary CTA : 1F: left coronary artery connected with pulmonary artery; $1 \mathrm{G}, \mathrm{H}$, I: there are abundant collateral vessels in the interventricular septum; $1 \mathrm{~J}$ : the posterior interventricular branch of the right crown was connected with the end of the anterior descending branch of the left crown; $1 \mathrm{~K}$ : the right coronal conus branch and the anterior branch of the right ventricle are connected with the branches of the left anterior descending branch.
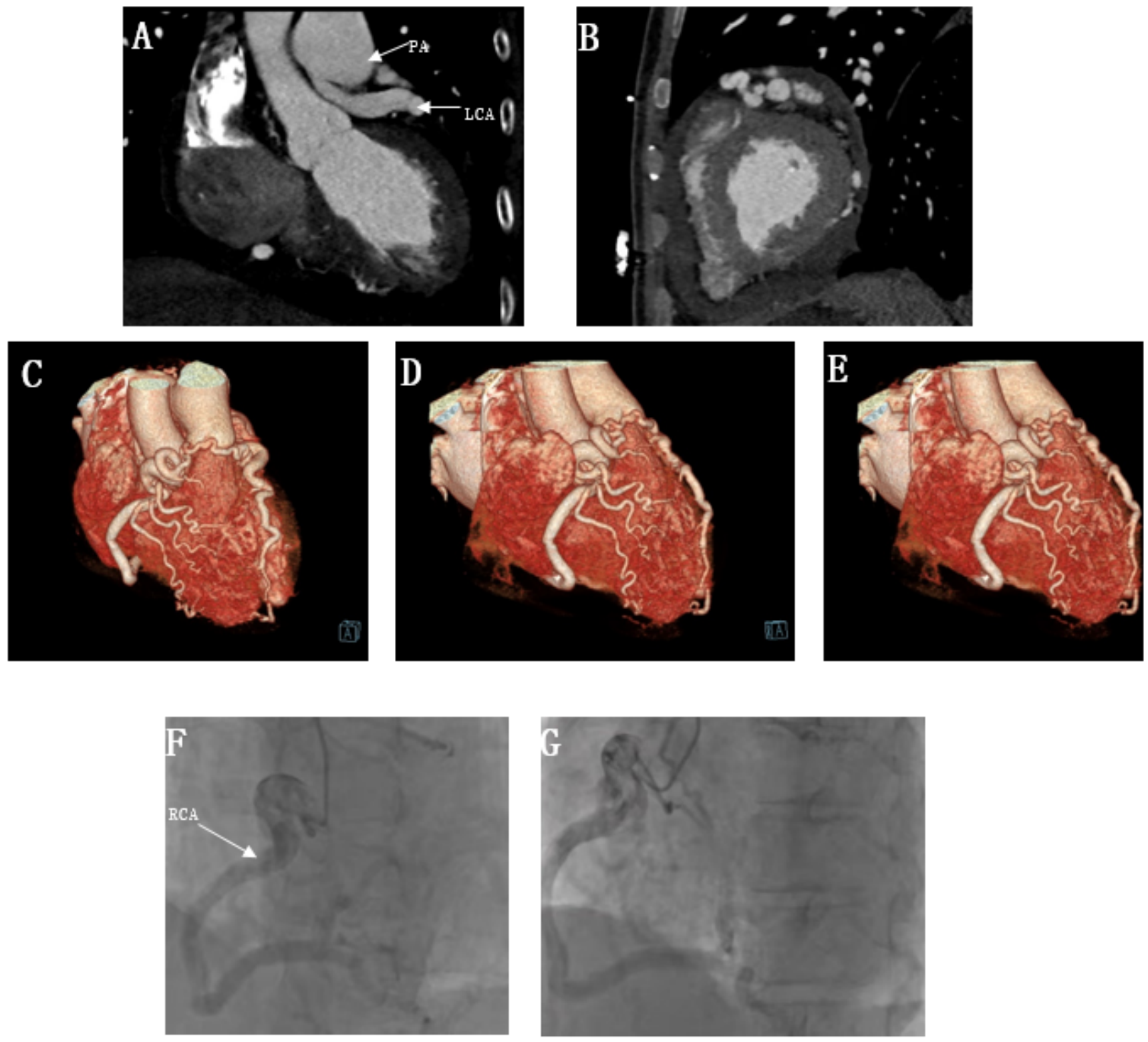

\section{Figure 2}

case 2.The coronary CTA indicated 2A: the left coronary artery was connected with the pulmonary artery; 2B: abundant collateral blood flow was seen in the interventricular septum. 2C, D, E: both the left and right 
coronary arteries were wide and malformed, and abundant collateral branches could be seen between them which formed by branches of right conus branch, anterior right ventricular branch and left anterior descending branch. case 2 . left coronary artery was absent and right coronary artery were wide malformation. 